\title{
Short- and long-term outcomes of laparoscopic surgery vs open surgery for transverse colon cancer: a retrospective multicenter study
}

This article was published in the following Dove Press journal:

OncoTargets and Therapy

15 April 2016

Number of times this article has been viewed

\author{
Jong Wan Kim' \\ Jeong Yeon Kim' \\ Byung Mo Kang ${ }^{2}$ \\ Bong Hwa Lee ${ }^{3}$ \\ Byung Chun $\mathrm{Kim}^{4}$ \\ Jun Ho Park ${ }^{5}$
}

'Department of Surgery, Dongtan Sacred Heart Hospital, College of Medicine, Hallym University, Hwaseong Si, ${ }^{2}$ Department of Surgery, Chuncheon Sacred Heart Hospital, College of Medicine, Hallym University, Chuncheon $\mathrm{Si}$, ${ }^{3}$ Department of Surgery, Hallym Sacred Heart Hospital, College of Medicine, Hallym University, Anyang Si, ${ }^{4}$ Department of Surgery, Kangnam Sacred Heart Hospital, College of Medicine, Hallym University, Seoul, ${ }^{5}$ Department of Surgery, Kangdong Sacred Heart Hospital, College of Medicine, Hallym University, Seoul, Republic of Korea
Correspondence: Jun Ho Park

Department of Surgery, Kangdong Sacred Heart Hospital, College of Medicine,

Hallym University, I 50 Seongan-ro, 445

Gil-I-dong, Gangdong-gu, I34-70I Seoul,

Republic of Korea

Tel +82 222242222

Fax +82 222241655

Email zooono@hanmail.net
Purpose: The purpose of the present study was to compare the perioperative and oncologic outcomes between laparoscopic surgery and open surgery for transverse colon cancer.

Patients and methods: We conducted a retrospective review of patients who underwent surgery for transverse colon cancer at six Hallym University-affiliated hospitals between January 2005 and June 2015. The perioperative outcomes and oncologic outcomes were compared between laparoscopic and open surgery.

Results: Of 226 patients with transverse colon cancer, 103 underwent laparoscopic surgery and 123 underwent open surgery. There were no differences in the patient characteristics between the two groups. Regarding perioperative outcomes, the operation time was significantly longer in the laparoscopic group than in the open group (267.3 vs 172.7 minutes, $P<0.001$ ), but the time to soft food intake ( 6.0 vs 6.6 days, $P=0.036)$ and the postoperative hospital stay ( $13.7 \mathrm{vs}$ 15.7 days, $P=0.018$ ) were shorter in the laparoscopic group. The number of harvested lymph nodes was lower in the laparoscopic group than in the open group (20.3 vs $24.3, P<0.001)$. The 5 -year overall survival $(90.8 \%$ vs $88.6 \%, P=0.540)$ and disease-free survival $(86.1 \%$ vs $78.9 \%, P=0.201$ ) rates were similar in both groups.

Conclusion: The present study showed that laparoscopic surgery is associated with several perioperative benefits and similar oncologic outcomes to open surgery for the resection of transverse colon cancer. Therefore, laparoscopic surgery offers a safe alternative to open surgery in patients with transverse colon cancer.

Keywords: transverse colon, colon cancer, laparoscopic surgery

\section{Introduction}

Laparoscopic colon surgery was first introduced in 1991, and laparoscopic surgery is now widely performed for resecting colorectal cancer. ${ }^{1}$ A Cochrane review showed that laparoscopic surgery was associated with similar oncologic outcomes and short-term benefits, including shorter hospital stay, less postoperative pain, improved cosmesis, and an earlier return to normal activity, as compared with open surgery.,3

However, previous randomized controlled trials excluded patients with transverse colon cancer because the surgical procedures used were dependent on the cancer location, the variable anatomy of the middle colic vessels, which demand excellent surgical skills, and the anatomical location of the transverse colon relative to major organs, especially the pancreas, spleen, and duodenum. ${ }^{4-12}$

In recent years, with accumulating experience of performing laparoscopic surgery, several studies have evaluated the safety and feasibility of laparoscopic resection of transverse colon cancer. ${ }^{13-19}$ However, the results of these studies should be interpreted 
carefully because few studies compared the clinical outcome of laparoscopic surgery for transverse colon cancer relative to those of other colon cancers, and these studies enrolled small number of patients, and the duration of follow-up were short.

The aims of the present study were to compare the perioperative, pathologic, and oncologic outcomes of laparoscopic surgery with those of open surgery for the resection of transverse colon cancer and to demonstrate the feasibility of laparoscopic surgery in this setting.

\section{Patients and methods}

We retrospectively reviewed the medical records of patients who underwent curative resection of transverse colon cancer at six Hallym University-affiliated hospitals (Kangnam Sacred Heart Hospital, Kangdong Sacred Heart Hospital, Chuncheon Sacred Heart Hospital, Dongtan Sacred Heart Hospital, Hangang Sacred Heart Hospital, and Hallym Sacred Heart Hospital) between January 2005 and February 2015. Patients were divided into two groups according to whether they underwent laparoscopic or open surgery. The study was approved by the Institutional Review Board of Dongtan Sacred Heart hospital (IRB 2015-268-I). As this was a retrospective study patient consent was not required.

Transverse colon cancer was defined as cancer located between the hepatic and splenic flexures. Patients with recurrent disease, familial adenomatous polyposis or hereditary nonpolyposis colorectal cancer, or stage 0 and IV cancer were excluded. Patients undergoing emergency surgery because of perforation and acute obstruction, and patients undergoing palliative resection were also excluded.

Patient demographic characteristics, operative variables, postoperative outcomes, histopathologic variables, and long-term oncologic outcomes were retrieved from the medical records. Patient demographics included age, sex, body mass index (BMI), American Society of Anesthesiologists score, prior surgical history, and cancer location. Operative variables included operation time, intraoperative transfusion, conversion, and procedure type. Postoperative outcomes included the time to flatus, time to soft food intake, postoperative hospital stay, complications, and the mortality rate within 30 days after surgery. The histopathologic report was examined to retrieve the following variables: histologic type of cancer, cancer size, proximal resection margin, distal resection margin, number of harvested lymph nodes, presence of positive lymph nodes, and disease stage (tumornode-metastasis [TNM] classification). Long-term oncologic outcomes included local recurrence, disease-free survival (DFS), and overall survival (OS).
The routine preoperative evaluations included physical examination, serum carcinoembryonic antigen (CEA) concentrations, abdominopelvic and chest computed tomography (CT), and colonoscopy. If necessary, magnetic resonance imaging and positron emission tomography-CT were performed for staging. Preoperative colonoscopic tattooing or clipping was performed if the cancer location was unclear.

Right hemicolectomy was performed for cancers located at the hepatic flexure, and left hemicolectomy was performed for cancers located at the splenic flexure. Transverse colectomy was performed for cancers located between the aforementioned two lesions. Right hemicolectomy was defined as ligation of the ileocolic, right colic, and the right branch of the middle colic vessels at their origins together with lymphadenectomy. If we needed to extend the right hemicolectomy, we ligated the origin of the middle colic vessels. Left hemicolectomy was defined as ligation of the left colic and the left branch of the middle colic vessels at their origins together with lymphadenectomy. If we needed to extend the left hemicolectomy, we ligated the origin of the middle colic vessels. Transverse colectomy was defined as ligation of the origin of the middle colic vessels together with lymphadenectomy. The choice of surgical approach was at the surgeon's discretion. Conversion to an open procedure was defined as an unplanned abdominal incision larger than necessary for specimen retrieval in the laparoscopic group.

Patients were followed up with physical examinations and measurement of serum CEA concentrations every 3 months for the first 2 years, and every 6 months thereafter until 5 years after surgery. Colonoscopy was repeated annually after surgery during the follow-up period. Abdominopelvic and chest CT scans were repeated every 6 months until 5 years. After 5 years, these evaluations were performed annually.

Local recurrence was defined as any recurrence within the anastomotic site. Systemic recurrence was defined as any recurrence outside the anastomotic site. DFS was defined as the time from surgery to disease recurrence or death. OS was defined as the time from surgery to death or the last follow-up date.

Categorical variables are presented as the number and percent of patients, and were analyzed using the $\chi^{2}$ test or Fisher's exact test. Continuous variables are presented as the mean and standard deviation and were compared using Student's $t$-test or the Mann-Whitney $U$ test. DFS and OS were analyzed using the Kaplan-Meier method and were compared using the log-rank test. $P$-values of $<0.05$ were considered statistically significant. 


\section{Results}

A total of 226 patients with transverse colon cancer were included in our study, of whom 122 (54.0\%) underwent open surgery and 104 (46.0\%) underwent laparoscopic surgery. The patient demographic characteristics are summarized in Table 1 . The mean age of the laparoscopic and open surgery groups was 65.6 and 62.8 years, respectively $(P=0.113)$. There were no significant differences in the proportion of males, BMI, American Society of Anesthesiologists score, and history of prior surgery. The cancer was located in the proximal transverse colon in 141 patients $(62.4 \%)$, the middle transverse colon in 31 patients $(13.7 \%)$, and the distal transverse colon in 54 patients $(23.9 \%)$; the site distribution was similar in both groups $(P=0.107)$.

The operative variables are shown in Table 2 . The mean operation time was longer in the laparoscopic group than in the open group (267.3 vs 172.7 minutes, respectively, $P<0.001)$, but the frequency of intraoperative transfusion was similar in both groups $(P=0.151)$. Transverse colectomy was more frequently performed in the open group (13.1\% vs $2.9 \%$, respectively), whereas left hemicolectomy was more frequently performed in the laparoscopic group (27.9\% vs $17.2 \%$, respectively). There were six (5.8\%) conversions from laparoscopic surgery to open surgery because of bleeding in two patients, severe adhesion in three patients, and a fixed tumor mass in one patient.

The postoperative outcomes are shown in Table 3. Although the time to flatus was similar in both groups, the time to soft food intake (6.0 vs 6.6 days, respectively, $P=0.036$ ) and the postoperative hospital stay (13.2 vs 15.7 days,

Table I Patient characteristics

\begin{tabular}{llll}
\hline Variables & $\begin{array}{l}\text { Open surgery } \\
(\mathbf{n}=\mathbf{I} 23)\end{array}$ & $\begin{array}{l}\text { Laparoscopic } \\
\text { surgery }(\mathbf{n}=103)\end{array}$ & P-value \\
\hline Age, years & $62.8(14.0)$ & $65.6(12.1)$ & 0.113 \\
Age $>60, \mathrm{n}(\%)$ & $91(74.6)$ & $83(79.8)$ & 0.353 \\
Male, n (\%) & $66(54.1)$ & $68(65.4)$ & 0.085 \\
BMI, kg/m ${ }^{2}$ & $23.1(3.1)$ & $23.5(4.0)$ & 0.399 \\
ASA, n (\%) & & & 0.548 \\
I & $20(16.4)$ & $20(19.4)$ & \\
2 & $80(65.6)$ & $58(56.3)$ & 0.354 \\
3 & $21(17.2)$ & $24(23.3)$ & 0.107 \\
$\quad 4$ & $1(0.8)$ & $1(1.0)$ & \\
Previous op history & $21(17.2)$ & $23(22.1)$ & \\
Tumor location & & & \\
$\quad$ Proximal & $74(60.7)$ & $67(64.4)$ & \\
Middle & $22(18.0)$ & $9(8.7)$ & \\
Distal & $26(21.3)$ & $28(26.9)$ & \\
\hline
\end{tabular}

Note: Data are presented as the number of patients (\%) or mean (standard deviation) unless otherwise stated.

Abbreviations: BMI, body mass index; ASA, American Society of Anesthesiologists; Op, operation.
Table 2 Operative outcome according to type of surgery

\begin{tabular}{|c|c|c|c|}
\hline Variables & $\begin{array}{l}\text { Open surgery } \\
(n=123)\end{array}$ & $\begin{array}{l}\text { Laparoscopic } \\
\text { surgery }(n=103)\end{array}$ & $P$-value \\
\hline Operation time, minutes & $172.7(54.0)$ & $267.3(74.8)$ & $<0.001$ \\
\hline Transfusion & $21(17.2)$ & $26(25.0)$ & 0.151 \\
\hline Procedures & & & 0.007 \\
\hline Right hemicolectomy & $85(69.7)$ & $72(69.2)$ & \\
\hline Transverse colectomy & $16(13.1)$ & $3(2.9)$ & \\
\hline Left hemicolectomy & $21(17.2)$ & $29(27.9)$ & \\
\hline Conversion & & $6(5.8)$ & \\
\hline Mortality, n (\%) & $0(0)$ & $0(0)$ & NA \\
\hline
\end{tabular}

Note: Data are presented as the number of patients (\%) or mean (standard deviation) unless otherwise stated.

Abbreviation: NA, not available.

respectively, $P=0.018$ ) were shorter in the laparoscopic group than in the open group. Complications were more frequent in the open group than in the laparoscopic group, although the difference was not statistically significant $(20.5 \%$ vs $11.5 \%$, respectively, $P=0.070$ ). There were no differences between the two groups in terms of wound complications, ileus, postoperative bleeding, anastomotic leakage, and intraabdominal abscess, but pulmonary complications were more frequent in the laparoscopic group than in the open group (5.7\% vs $0 \%, P=0.016)$. There were no postoperative deaths in either group.

Cancer size, proximal and distal resection margins, histologic type, and TNM stage were similar in both groups. The number of harvested lymph nodes was significantly greater in the open group than in the laparoscopic group (24.3 vs 20.3, respectively, $P<0.001$ ). According to the TNM classification, patients in the laparoscopic group tended to have earlier-stage disease compared with patients in the open group, although the difference was not statistically significant $(P=0.068)$ (Table 4).

Table 3 Postoperative outcome

\begin{tabular}{|c|c|c|c|}
\hline Variables & $\begin{array}{l}\text { Open surgery } \\
(n=123)\end{array}$ & $\begin{array}{l}\text { Laparoscopic } \\
\text { surgery }(n=103)\end{array}$ & $P$-value \\
\hline Time to flatus, days & $4.5(1.7)$ & $4.5(1.2)$ & 0.716 \\
\hline $\begin{array}{l}\text { Time to soft food intake, } \\
\text { days }\end{array}$ & $6.6(2.2)$ & $6.0(1.6)$ & 0.036 \\
\hline Duration of POD, days & I5.7 (9.7) & $13.2(5.6)$ & 0.018 \\
\hline Complications, n (\%) & $25(20.5)$ & $12(11.5)$ & 0.070 \\
\hline Wound & $2(1.6)$ & $2(1.9)$ & 1.000 \\
\hline lleus & $8(6.6)$ & $6(5.8)$ & 1.000 \\
\hline Pulmonary & $7(5.7)$ & $0(0)$ & 0.016 \\
\hline Postoperative bleeding & $7(5.7)$ & $2(1.9)$ & 0.184 \\
\hline Anastomotic leakage & $2(2.5)$ & I (I.0) & 0.626 \\
\hline Intrabdominal abscess & $2(1.6)$ & $3(2.9)$ & 0.663 \\
\hline Complications $\geq 2$ & $4(3.3)$ & $2(1.9)$ & 0.689 \\
\hline
\end{tabular}

Note: Data are presented as the number of patients (\%) or median (standard deviation) unless otherwise stated.

Abbreviations: POD, postoperative days; n, number. 
Table 4 Pathologic outcome

\begin{tabular}{|c|c|c|c|}
\hline Variables & $\begin{array}{l}\text { Open surgery } \\
(n=123)\end{array}$ & $\begin{array}{l}\text { Laparoscopic } \\
\text { surgery }(n=103)\end{array}$ & $P$-value \\
\hline Tumor size, cm & $5.3(3.0)$ & $5.0(2.6)$ & 0.328 \\
\hline PRM, cm & $16.8(10.1)$ & $15.7(9.5)$ & 0.413 \\
\hline DRM, cm & $10.3(6.7)$ & $10.5(12.7)$ & 0.847 \\
\hline No of harvested LN & $24.3(15.5)$ & $20.3(9.8)$ & 0.019 \\
\hline $\begin{array}{l}\text { Proportion of } \\
\text { positive LN, n (\%) }\end{array}$ & $47(38.5)$ & $32(30.8)$ & 0.223 \\
\hline TNM stage, n (\%) & & & 0.068 \\
\hline I & $18(14.6)$ & $26(25.2)$ & \\
\hline II & $58(47.5)$ & $45(43.3)$ & \\
\hline III & 47 (38.5) & $32(30.8)$ & \\
\hline Histologic type, n (\%) & & & 0.635 \\
\hline Well & 45 (36.9) & $45(43.3)$ & \\
\hline Moderate & $57(46.7)$ & $45(43.3)$ & \\
\hline Poor & $10(8.2)$ & $5(4.8)$ & \\
\hline Mucinous & II (8.9) & $8(7.8)$ & \\
\hline
\end{tabular}

Note: Data are presented as the number of patients (\%) or median (standard deviation) unless otherwise stated.

Abbreviations: PRM, proximal resection margin; DRM, distal resection margin; No, number; LN, lymph node; TNM, tumor-node-metastasis.

The mean duration of follow-up was 46 and 54 months in the laparoscopic and open groups, respectively. The 5-year OS rates were $90.8 \%$ and $88.6 \%$ in the laparoscopic and open groups, respectively $(P=0.540)$ (Figure 1A). Subgroup analysis of stage II and III transverse colon cancer patients revealed that the 5-year OS was not significantly different between the laparoscopic and open groups in stage II patients ( $81.3 \%$ vs $86.1 \%, P=0.817)$ or stage III patients $(81.2 \%$ vs $68.1 \%, P=0.301$ ) (Figure 1B and $\mathrm{C}$ ). The 5-year DFS rates were $86.1 \%$ and $78.9 \%$ in the laparoscopic and open groups, respectively $(P=0.201)$ (Figure $2 \mathrm{~A})$. In a subgroup analysis of stage II and III patients, the 5-year DFS was not significantly different between the laparoscopic and open groups in stage II patients $(65.7 \%$ vs $67.4 \%, P=0.471)$ or stage III patients (69.2\% vs 62.8\%, $P=0.314$ ) (Figure $2 \mathrm{~B}$ and C). Disease recurrence occurred in a similar number of patients in the laparoscopic and open groups $(12.5 \%$ vs $18.0 \%, P=0.252)$. The most common site of recurrence was the peritoneum, followed by the liver, lung, small bowel, abdominal lymph nodes, rectum, stomach, brain, and incision site (Table 5).

\section{Discussion}

Several randomized controlled trials have demonstrated that laparoscopic surgery provides favorable short-term outcomes and similar long-term oncologic outcomes compared with open surgery for the resection of colon cancer. ${ }^{4-12}$ However, transverse colon cancer was excluded from the previous trials for several reasons. First, the type of surgical procedure and the extent of lymph node dissection vary according to the cancer's location. Second, laparoscopic surgery for
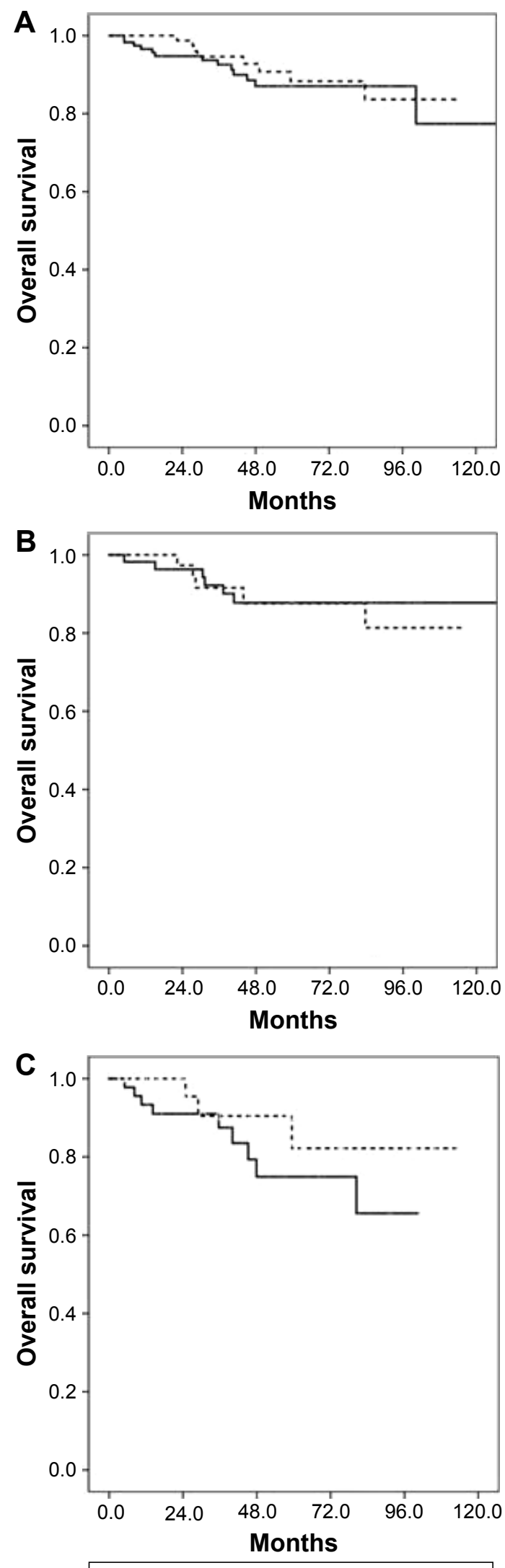

Open surgery - Laparoscopic surgery

Figure I Comparison of 5-year overall survival between the laparoscopic and open groups in all patients ( $\mathbf{A} ; \mathbf{9 0 . 8 \%}$ vs $88.6 \%, P=0.540)$, patients with stage II transverse colon cancer (B; $81.3 \%$ vs $86.1 \%, P=0.817$ ), and patients with stage III transverse colon cancer (C; $81.2 \%$ vs $68.1 \%, P=0.301$ ). 

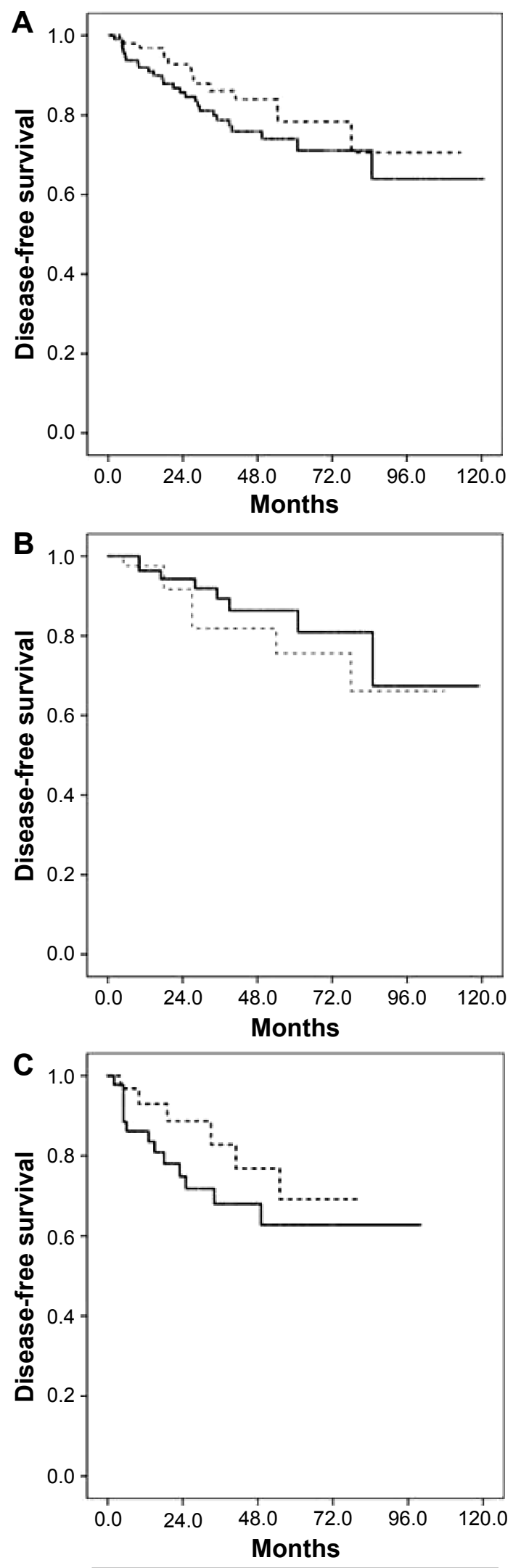

ח Open surgery Laparoscopic surgery

Figure 2 Comparison of 5-year disease-free survival between the laparoscopic and open groups in all patients (A; $86.1 \%$ vs $78.9 \%, P=0.201$ ), patients with stage II transverse colon cancer (B; $65.7 \%$ vs $67.4 \%, P=0.47 \mathrm{I}$ ), and patients with stage III colon cancer (C; $69.2 \%$ vs $62.8 \%, P=0.314)$.
Table 5 Kaplan-Meier survival curve comparing local recurrence, DFS and OS

\begin{tabular}{|c|c|c|c|}
\hline Variables & $\begin{array}{l}\text { Open surgery } \\
(n=123)\end{array}$ & $\begin{array}{l}\text { Laparoscopic } \\
\text { surgery }(n=103)\end{array}$ & P-value \\
\hline \multicolumn{4}{|l|}{ 5-year OS } \\
\hline Stage all & $88.6 \%$ & $90.8 \%$ & 0.540 \\
\hline Stage II & $86.1 \%$ & $81.3 \%$ & 0.817 \\
\hline Stage III & $68.1 \%$ & $81.2 \%$ & 0.301 \\
\hline \multicolumn{4}{|l|}{ 5-year DFS } \\
\hline Stage all & $78.9 \%$ & $86.1 \%$ & 0.201 \\
\hline Stage II & $67.4 \%$ & $65.7 \%$ & 0.471 \\
\hline Stage III & $62.8 \%$ & $69.2 \%$ & 0.314 \\
\hline Recurrence, n (\%) & $22(18.0)$ & $13(12.5)$ & 0.252 \\
\hline Anastomotic site & 0 & I & \\
\hline Peritoneum & 5 & 4 & \\
\hline Liver & 5 & 3 & \\
\hline Lung & 3 & 4 & \\
\hline Small bowel & 4 & 2 & \\
\hline Abdominal lymph node & 3 & I & \\
\hline Rectum & 2 & 0 & \\
\hline Stomach & 2 & 0 & \\
\hline Brain & I & 0 & \\
\hline Incision site & 0 & I & \\
\hline Ovary & 1 & 0 & \\
\hline Recurrent site $\geq 2$ & 4 & 5 & \\
\hline
\end{tabular}

Abbreviations: DFS, disease-free survival; OS, overall survival.

transverse colon cancer requires excellent surgical skills owing to the technical difficulty of lymph node dissection around the middle colic vessels, and the anatomical variations of the middle colic vessels. ${ }^{15,19,20}$ Mobilization of the transverse colon involves dissection around critical organs such as the duodenum, pancreas, and spleen, and the base of the mesenteric vessels, which may result in serious complications if dissection occurs along the wrong plane. ${ }^{21}$ Finally, surgeons have limited experience of treating transverse colon cancer because its accounts for only $\sim 10 \%$ of all colon cancers. ${ }^{22-24}$

With accumulating experience of laparoscopic surgery and continued developments in laparoscopic instruments, several studies have examined the safety and feasibility of laparoscopic surgery for resecting transverse colon cancer. ${ }^{13-19}$ In addition, several studies have reported the longterm oncologic outcomes of laparoscopic surgery. ${ }^{25-28}$

The present study reported that the time to soft diet and the postoperative hospital stay were significantly shorter in the laparoscopic group than in the open group. These results are consistent with those of previous studies. ${ }^{13-15,17,29}$

The postoperative complication rate was not significantly different between the two groups in prior studies, even though laparoscopic surgery requires excellent surgical skills for resecting transverse colon cancer. ${ }^{13-17,25-28}$ Nakashima et $\mathrm{a}^{29}$ reported that the postoperative complication rate was 
significantly lower in the laparoscopic group than in the open group ( $6 \%$ vs $36 \%, P<0.01$ ). In the present study, the complication rate tended to be lower in the laparoscopic group ( $11.5 \%$ vs $20.5 \%, P=0.070$ ). Of note, the rate of pulmonary complications was significantly lower in the laparoscopic group than in the open group ( $0 \%$ vs $5.7 \%, P=0.016$ ), which partly explains the observed difference in the overall complication rate between the two groups.

In the present study, the operation time was much longer in laparoscopic group than in the open group, as in previous studies. ${ }^{13-17,19}$ In addition, previous studies reported that operation time for laparoscopic surgery was longer for procedures involving the transverse colon than for other sites of the colon. ${ }^{14,18}$ These results may reflect the difficulty of performing laparoscopic surgery for transverse colon cancer and the need for extensive resection.

The principle for colon cancer surgery is en bloc resection of the colon to leave clear proximal and distal margins. In the present study, the cancer size, proximal margin, and distal margin were similar in both groups. However, the number of harvested lymph nodes was greater in the open group than in the laparoscopic group (24.3 vs 20.3, $P=0.019$ ). Akiyoshi et $\mathrm{al}^{14}$ also reported a greater number of harvested lymph nodes in their open resection group than in their laparoscopic resection group ( $23 \mathrm{vs} 17, P<0.01$ ). A systematic review demonstrated that the number of harvested lymph nodes is associated with survival and recommended that the surgeon should harvest at least 12 lymph nodes for adequate sampling. ${ }^{30}$ The mean number of harvested lymph nodes in the laparoscopic group was 20, which exceeds the recommended number of lymph node for adequate sampling. ${ }^{30}$

In previous studies, the 5-year OS ranged from $61 \%$ to $94.3 \%$ and from $59 \%$ to $88.6 \%$ in the laparoscopic and open groups, ${ }^{25-28}$ while 5-year DFS ranged from $70.5 \%$ to $80.4 \%$ and from $66.7 \%$ to $85.7 \%$, respectively. ${ }^{25-28}$ In the present study, the 5-year OS and DFS were $90.8 \%$ and $86.1 \%$, respectively, in the laparoscopic group, and $88.6 \%$ and $78.9 \%$, respectively, in the open group, which are within the ranges reported in the previous studies. Furthermore, the present study showed that the oncologic outcomes of laparoscopic surgery were comparable with those of open surgery in terms of the 5-year OS and DFS $(P=0.540$ and $P=0.201$, respectively).

Laparoscopic surgery for the resection of transverse colon cancer by experienced surgeons is associated with favorable short-term outcomes, especially faster recovery and shorter hospital stay, and equivalent long-term outcomes, including 5-year OS and DFS, relative to open surgery. These results demonstrate that laparoscopic surgery is an acceptable method for resecting transverse colon cancer.

The present study had several limitations. First, this study was performed retrospectively, which may introduce selection bias. In particular, the surgeons tended to perform laparoscopic surgery in patients with early-stage transverse colon cancer, whereas open surgery was preferred for advanced-stage cancer $(P=0.068)$. Second, although the number of laparoscopic surgical procedures for resecting transverse colon in our study $(>100)$ was greater than that in previous studies, ${ }^{13-16,19,25-27}$ the low incidence of transverse colon cancer limits the opportunity for prospective randomized clinical trials, which are needed to establish definitive conclusions. Despite these limitations, we believe that our results provide valuable support for laparoscopic resection of transverse colon cancer. A larger scaled, prospective, randomized controlled study is needed to confirm the safety and efficacy of laparoscopic resection of transverse colon cancer.

\section{Conclusion}

The present study has shown that laparoscopic surgery is associated with several perioperative benefits and similar oncologic outcomes relative to open surgery for the resection of transverse colon cancer. Therefore, we believe that laparoscopic surgery can be safely performed for the resection of transverse colon cancer.

\section{Acknowledgment}

This research was supported by Hallym University Research Fund 2015 (HURF-2015-48).

\section{Disclosure}

The authors report no conflicts of interest in this work.

\section{References}

1. Jacobs M, Verdeja JC, Goldstein HS. Minimally invasive colon resection (laparoscopic colectomy). Surg Laparosc Endosc. 1991;1(3):144-150.

2. Schwenk W, Haase O, Neudecker J, Muller JM. Short term benefits for laparoscopic colorectal resection. Cochrane Database Syst Rev. 2005; (3):CD003145.

3. Kuhry E, Schwenk WF, Gaupset R, Romild U, Bonjer HJ. Long-term results of laparoscopic colorectal cancer resection. Cochrane Database Syst Rev. 2008;(2):CD003432.

4. Guillou PJ, Quirke P, Thorpe H, et al. Short-term endpoints of conventional versus laparoscopic-assisted surgery in patients with colorectal cancer (MRC CLASICC trial): multicentre, randomised controlled trial. Lancet. 2005;365(9472):1718-1726.

5. Clinical Outcomes of Surgical Therapy Study Group. A comparison of laparoscopically assisted and open colectomy for colon cancer. $N \mathrm{Engl}$ J Med. 2004;350(20):2050-2059.

6. Lacy AM, Garcia-Valdecasas JC, Delgado S, et al. Laparoscopy-assisted colectomy versus open colectomy for treatment of non-metastatic colon cancer: a randomised trial. Lancet. 2002;359(9325):2224-2229. 
7. Braga M, Vignali A, Zuliani W, Frasson M, Di Serio C, Di Carlo V. Laparoscopic versus open colorectal surgery: cost-benefit analysis in a single-center randomized trial. Ann Surg. 2005;242(6):890-895.

8. Jayne DG, Guillou PJ, Thorpe H, et al. Randomized trial of laparoscopicassisted resection of colorectal carcinoma: 3-year results of the UK MRC CLASICC Trial Group. J Clin Oncol. 2007;25(21):3061-3068.

9. Fleshman J, Sargent DJ, Green E, et al. Laparoscopic colectomy for cancer is not inferior to open surgery based on 5-year data from the COST Study Group trial. Ann Surg. 2007;246(4):655-662.

10. Jayne DG, Thorpe HC, Copeland J, Quirke P, Brown JM, Guillou PJ. Five-year follow-up of the Medical Research Council CLASICC trial of laparoscopically assisted versus open surgery for colorectal cancer. Br J Surg. 2010;97(11):1638-1645.

11. Buunen M, Veldkamp R, Hop WC, et al. Survival after laparoscopic surgery versus open surgery for colon cancer: long-term outcome of a randomised clinical trial. Lancet Oncol. 2009;10(1):44-52.

12. Veldkamp R, Kuhry E, Hop WC, et al. Laparoscopic surgery versus open surgery for colon cancer: short-term outcomes of a randomised trial. Lancet Oncol. 2005;6(7):477-484.

13. Fernandez-Cebrian JM, Gil Yonte P, Jimenez-Toscano M, Vega L, Ochando F. Laparoscopic colectomy for transverse colon carcinoma: a surgical challenge but oncologically feasible. Colorectal Dis. 2013; 15(2):e79-e83.

14. Akiyoshi T, Kuroyanagi H, Fujimoto Y, et al. Short-term outcomes of laparoscopic colectomy for transverse colon cancer. J Gastrointest Surg. 2010;14(5):818-823.

15. Kim HJ, Lee IK, Lee YS, et al. A comparative study on the short-term clinicopathologic outcomes of laparoscopic surgery versus conventional open surgery for transverse colon cancer. Surg Endosc. 2009;23(8): 1812-1817.

16. Zmora O, Bar-Dayan A, Khaikin M, et al. Laparoscopic colectomy for transverse colon carcinoma. Tech Coloproctol. 2010;14(1):25-30.

17. Yamamoto M, Okuda J, Tanaka K, Kondo K, Tanigawa N, Uchiyama K. Clinical outcomes of laparoscopic surgery for advanced transverse and descending colon cancer: a single-center experience. Surg Endosc. 2012; 26(6):1566-1572.

18. Hirasaki Y, Fukunaga M, Sugano M, Nagakari K, Yoshikawa S, Ouchi M. Short- and long-term results of laparoscopic surgery for transverse colon cancer. Surg Today. 2014;44(7):1266-1272.
19. Schlachta CM, Mamazza J, Poulin EC. Are transverse colon cancers suitable for laparoscopic resection? Surg Endosc. 2007;21(3):396-399.

20. Yamaguchi S, Kuroyanagi H, Milsom JW, Sim R, Shimada H. Venous anatomy of the right colon: precise structure of the major veins and gastrocolic trunk in 58 cadavers. Dis Colon Rectum. 2002;45(10): 1337-1340.

21. Jamali FR, Soweid AM, Dimassi H, Bailey C, Leroy J, Marescaux J. Evaluating the degree of difficulty of laparoscopic colorectal surgery. Arch Surg. 2008;143(8):762-767.

22. Wray CM, Ziogas A, Hinojosa MW, Le H, Stamos MJ, Zell JA. Tumor subsite location within the colon is prognostic for survival after colon cancer diagnosis. Dis Colon Rectum. 2009;52(8):1359-1366.

23. Sjo OH, Lunde OC, Nygaard K, Sandvik L, Nesbakken A. Tumour location is a prognostic factor for survival in colonic cancer patients. Colorectal Dis. 2008;10(1):33-40.

24. Hayne D, Brown RS, McCormack M, Quinn MJ, Payne HA, Babb P. Current trends in colorectal cancer: site, incidence, mortality and survival in England and Wales. Clin Oncol. 2001;13(6):448-452.

25. Kim WR, Baek SJ, Kim CW, et al. Comparative study of oncologic outcomes for laparoscopic vs open surgery in transverse colon cancer. Ann Surg Treat Res. 2014;86(1):28-34.

26. Zhao L, Wang Y, Liu H, et al. Long-term outcomes of laparoscopic surgery for advanced transverse colon cancer. J Gastrointest Surg. 2014; 18(5):1003-1009.

27. Mistrangelo M, Allaix ME, Cassoni P, Giraudo G, Arolfo S, Morino M. Laparoscopic versus open resection for transverse colon cancer. Surg Endosc. 2015;29(8):2196-2202.

28. Zeng WG, Liu MJ, Zhou ZX, et al. Outcome of laparoscopic versus open resection for transverse colon cancer. J Gastrointest Surg. 2015;19(10) 1869-1874

29. Nakashima M, Akiyoshi T, Ueno M, et al. Colon cancer in the splenic flexure: comparison of short-term outcomes of laparoscopic and open colectomy. Surg Laparosc Endosc Percutan Tech. 2011;21(6):415-418.

30. Chang GJ, Rodriguez-Bigas MA, Skibber JM, Moyer VA. Lymph node evaluation and survival after curative resection of colon cancer: systematic review. J Natl Cancer Inst. 2007;99(6):433-441.
OncoTargets and Therapy

\section{Publish your work in this journal}

OncoTargets and Therapy is an international, peer-reviewed, open access journal focusing on the pathological basis of all cancers, potential targets for therapy and treatment protocols employed to improve the management of cancer patients. The journal also focuses on the impact of management programs and new therapeutic agents and protocols on

\section{Dovepress}

patient perspectives such as quality of life, adherence and satisfaction. The manuscript management system is completely online and includes a very quick and fair peer-review system, which is all easy to use. Visit http://www.dovepress.com/testimonials.php to read real quotes from published authors. 